\title{
Kinderbetreuung im Cono Sur
}

\author{
Die Fälle Chile und Uruguay ${ }^{1}$
}

\author{
William Daniel VERA² und Carina DE LA BARRA ${ }^{3}$
}

\section{Pontificia Universidad Católica de Valparaíso}

Care-Arbeiten werden hauptsächlich von Frauen geleistet, ohne dass diese dabei eine ausreichende Unterstützung seitens der öffentlichen Politik erhalten. Resultat davon ist eine ungleiche Verteilung der Aufgaben, der Mittel und des Arbeitsaufwands zwischen den Geschlechtern. Diese Arbeitsteilung beeinflusst die sozialen Möglichkeiten der Frauen und trägt dazu bei, die Ungleichheiten zwischen den Geschlechtern zu vertiefen. In Lateinamerika ist in den letzten Jahren aufgrund des demografischen Wandels, der Veränderungen in der Familienstruktur und -organisation sowie der zunehmenden Einbindung der Frauen in den Arbeitsmarkt eine Krise des traditionellen Care-Modells zu beobachten (Ullmann, Maldonado Valera und Nieves Rico, 2014).

Angeregt durch den von Esping-Andersen (1990) entwickelten Ansatz der Wohlfahrtsregime führten Daly und Lewis (2011) den Begriff Social Care ein, um auf die Aufteilung der Verantwortung der verschiedenen Bereiche - Staat, Markt, Familie und Gemeinschaft - in Bezug auf Beschäftigung, Zuständigkeiten und Kosten der Betreuungsarbeiten hinzuweisen. Dieser Ansatz untersucht, inwiefern bestimmte Organisationstypen und Formen der Mittelbereitstellung die Funktion der Aufgaben im Bereich der Familie unterstützen oder nicht. Dabei werden zwei Begriffe herangezogen: der des Familialismus, der den Verbleib der Pflege und Betreuung im familiären Bereich bezeichnet; und der des De-Familialismus, der sich auf die Entlastung der Familie von diesen Aufgaben bezieht (Esping-Andersen, 2000). Komplementär dazu unterscheidet Leitner (2003) vier Varianten des Familialismus, um zu bestimmen, welche Art von Familialismus von einem bestimmten Wohlfahrtsregime gefördert wird: expliziter Familialismus, optionaler Familialismus, impliziter Familialismus und DeFamilialismus. Diese vier Varianten decken das traditionelle Modell ab, in der die zentrale Verantwortungsrolle der Familie für die Pflege und Betreuung gefördert wird, bis hin zur Variante, die die Übertragung der Aufgaben auf andere Wohlfahrtsbereiche zulässt. Jede Familialismus-Variante fördert zudem in der Bevölkerung bestimmte Formen von Geschlechtergerechtigkeit und sozialer Gerechtigkeit, indem sie die Ungleichheiten entweder verstärkt oder

\footnotetext{
${ }^{1}$ Dieser Artikel ist Bestandteil der Ergebnisse des Projekts CONICYT-PAI No 821320016, finanziert durch die Nationale Kommission für Wissenschaft und Technologie Chile (Comisión Nacional de Ciencia y Tecnología de Chile)

2 William Daniel Vera, PhD.(occ.), Pontificia Universidad Católica de Valparaíso Chile, Valparaíso Chile, william.vera@ucv.cl. Assoziierter Forscher der Escuela de Trabajo Social, Pontificia Universidad Católica de Valparaíso.

${ }^{3}$ Carina de la Barra, licenciada (occ.), Pontificia Universidad Católica de Valparaíso Chile, Valparaíso Chile, carina.delabarra@gmail.com. Forschungsassistentin, Escuela de Trabajo Social, Pontificia Universidad Católica de Valparaíso.
} 
verringert. Gemäss Blofield und Martínez Franzoni (2015) drückt sich die Geschlechtergerechtigkeit in drei Idealtypen aus: maternalistisch geprägt, gemässigt maternalistisch geprägt und die gemeinsame Verantwortung zwischen den Geschlechtern betonend. Die soziale Gerechtigkeit nimmt drei verschiedene Ausprägungen an: bedarfsorientiert, kontributiv bzw. korporatistisch sowie staatsbürgerlich bzw. universell-rechtlich (Blofield und Martínez Franzoni, 2015).

In Lateinamerika weisen Länder wie Chile und Uruguay fortschrittliche Modelle sozialer Sicherheit auf, die sich jedoch hinsichtlich ihres jeweiligen gesellschaftlichen Solidaritätstypus unterscheiden. Ein Modell ist liberal und unterstützt bedarfsorientierte sozialpolitische Massnahmen, während das andere eher korporatistisch geprägt ist und universalistische Massnahmen vorsieht. Beide Länder weisen Veränderungen in ihren soziodemografischen Strukturen auf. Hierzu gehören eine deutliche Abnahme der Fertilitätsraten, eine leichte Zunahme des Anteils von Frauen auf dem Arbeitsmarkt sowie Veränderungen der Familienstrukturen. Während der Abhängigkeitsquotient in der Kinderbetreuung zurückgeht - was sich durch den schrumpfenden Anteil Kinder in der Bevölkerung erklärt -, ist gleichzeitig zu beobachten, dass sich Frauen weniger ausschliesslich der unbezahlten Hausarbeit widmen (CEPAL, 2015; INE, 2013, 2015).

Chile weist einen gemischten Charakter der ausserhäuslichen Kinderbetreuung auf, da sowohl Staat als auch Markt Dienstleistungen erbringen. Auf staatlicher Seite findet sich das 2006 gegründete Programm Chile Crece Contigo, das kostenlose Betreuungsangebote für Kinder im Alter von bis zu vier Jahren in öffentlichen Kindertagesstätten und Kindergärten vorsieht. Dieses Programm richtet sich an Familien aus den ersten drei Einkommensquintilen, in denen Mütter formell oder informell beschäftigt sind, auf Arbeitssuche sind oder sich in Ausbildung befinden (Staab, 2013). Unternehmen, die 20 oder mehr Frauen beschäftigen, stellen eine weitere Betreuungsform für Kinder im Alter von unter zwei Jahren bereit (Gesetz im Arbeitsrecht zur „Sala Cuna“, Código del Trabajo) (DT, 2015). Gemäss dieser arbeitsrechtlichen Vorschrift ist der Arbeitgeber verpflichtet, einer angestellten Mutter Betreuungsleistungen bereitzustellen oder zu bezahlen. Bei der Erziehung auf der Vorprimarstufe für Kinder im Alter von vier und fünf Jahren verfügt das Bildungssystem sowohl über ein staatliches Angebot als auch über subventionierte und nicht-subventionierte Privateinrichtungen (Staab, 2013).

Uruguay hat vor kurzem ein neues auf Rechten basiertes, universelles staatliches Pflegeund Betreuungssystem (Sistema Nacional Integrado de Cuidados, SNIC) eingeführt (Batthyány, 2015). Die ausserhäuslichen Betreuungsleistungen erfolgen in frühkindlichen Betreuungs- (für Kinder im Alter von bis zu zwei Jahren) und Vorschuleinrichtungen (Kinder im Alter von drei bis fünf Jahren). Das an Kleinkinder gerichtete private Angebot setzt sich aus bezahlten, vom Bildungs- und Kulturministerium (Ministerio de Educación y Cultura, $M E C)$ regulierten Vorschuleinrichtungen sowie den Schulen und Kindergärten zusammen, die durch die Erziehungskommission der Vorprimar- und Primarstufe (Consejo de Educación Inicial y Primaria, CEIP) zugelassen werden, einer Behörde der Nationalen Verwaltung des öffentlichen Schulwesens (Administración Nacional de Educación Pública, ANEP). Das staatliche Angebot erfolgt über die öffentlichen CEIP-ANEP-Kindergärten, die Kinder- und Familienzentren (Centros de Atención en Infancia y la Familia, CAIF), die zwar privat geführt, 
jedoch vom uruguayischen Kinder- und Jugendinstitut (Instituto del Niño y del Adolescente del Uruguay, INAU) finanziert und überwacht werden, sowie die direkt vom INAU geleitete Kindertagesbetreuung. Für Vier- bis Fünfjährige ist das Angebot an Care-Leistungen über öffentliche Schulen und Kindergärten und in kleinerem Masse über private, durch die ANEP zugelassene Schulen und Kindergärten organisiert (MIDES, 2014).

\begin{tabular}{|c|c|c|c|c|}
\hline & & Chile & Uruguay & $\begin{array}{c}\text { Differenz } \\
\text { zwischen bei- } \\
\text { den Ländern } \\
\end{array}$ \\
\hline \multirow{2}{*}{$\begin{array}{l}\text { Ausserhäusliche Be- } \\
\text { treuung } \\
\text { (Versorgungsgrad) } \\
\end{array}$} & $0-3$ Jahre & $28.4 \%$ & $34.6 \%$ & $6.2 \%$ \\
\hline & 4-5 Jahre & $87.4 \%$ & $94.4 \%$ & $7.0 \%$ \\
\hline \multirow[t]{2}{*}{$\begin{array}{l}\text { Häusliche Betreuung } \\
\text { (Beteiligung) }\end{array}$} & $\begin{array}{l}\text { Frauen (15 Jahre oder älter), } \\
\text { die sich ausschliesslich der } \\
\text { Hausarbeit widmen und im } \\
\text { Haushalt eine oder mehrere } \\
\text { minderjährige Personen be- } \\
\text { treuen. }\end{array}$ & $18.1 \%$ & $23 \%$ & $4.9 \%$ \\
\hline & Als Haushaltshilfe Angestellte & $8.6 \%$ & $9.6 \%$ & $1.0 \%$ \\
\hline
\end{tabular}

Tabelle 1: Bereitstellung ausserhäuslicher und häuslicher Kinderbetreuung, 2013. Quelle: Eigene Berechnung anhand der Daten von CEPALSTAT

Die häusliche Kinderbetreuung erfolgt in Form von bezahlter und unbezahlter Hausarbeit. Für den ersten Fall verfügen beide Länder über kürzlich verabschiedete Arbeitsgesetze, die die Arbeitsbedingungen, -zeiten und -gehälter von Haushaltshilfen an jene der im privaten Sektor beschäftigten Lohnarbeiter angleichen (in Uruguay das Gesetz Nr. 18.065 von 2006; in Chile das Gesetz Nr. 20.786 von 2014 (MINTRAB, 2014; MTSS, 2006). Unbezahlte Hausarbeit wird in keinem der beiden Länder per Gesetz anerkannt. Tabelle 1 fasst für beide Länder den Versorgungsgrad und die Beteiligung an den ausserhäuslichen und häuslichen CareLeistungen für 2013 zusammen.

Bezüglich des Betreuungsurlaubs reformierten beide Länder vor Kurzem ihre Gesetzgebung. Mit dem Gesetz NR. 20.545 von 2011 (MIDEPLAN, 2006) hat Chile die Freistellungsmöglichkeiten für die Kinderbetreuung erweitert. Frauen haben das Recht, sechs Wochen vor und 24 Wochen nach der Geburt freigestellt zu werden. Männer bekommen nach der Geburt einen Vaterschaftsurlaub von fünf Tagen zugesprochen. Die neu geschaffene Elternzeit ermöglicht es dem Mann, ab der 13.Woche der Elternzeit bis zu sechs Wochen Urlaub zu nehmen. Bei Halbtagsarbeit kann die Frau ausserdem ihre Elternzeit von 12 auf 18 Wochen verlängern. Der Mann kann 12 der 18 Wochen in Anspruch nehmen, wenn er halbtags arbeitet (Lupica, 2015). In Uruguay gilt das Gesetz Nr. 19.161 von 2013 (MTSS, 2013), das einen Mutterschaftsurlaub von 14 Wochen, einen Vaterschaftsurlaub, der sich bis 2016 graduell von drei auf 13 Tage erhöht, sowie eine Elternzeit vorsieht, die es ermöglicht, dass beide Eltern das 
Kind bis zum Alter von sechs Monaten gemeinsam betreuen können wenn sie halbtags arbeiten. Der Elternurlaub kann zwischen Vater und Mutter aufgeteilt werden (Batthyány, Genta und Perrotta, 2015).

Tabelle 2 (vgl. folgende Seite) vergleicht die Kinderbetreuungsmodelle des jeweiligen Care-Regimes. Beide Länder weisen einen geringen Versorgungsgrad bei ausserhäuslichen Dienstleistungen für Kinder im Alter von bis drei Jahren auf, sind geprägt durch eine geringe De-Familiarisierung und eine hinsichtlich der Geschlechtergerechtigkeit maternalistische Ausrichtung. Chile weist eine soziale Gerechtigkeit auf, die sich in drei Status segmentiert: bedarfsorientiert für untere Schichten sowie untere Mittelschichten (Chile Crece Contigo), kontributiv für lohnbeschäftigte Frauen (Recht auf Sala Cuna) und marktförmig für einkommensstärkere Haushalte. Uruguay weist eine segmentierte Struktur auf, die in zwei Status geordnet ist: bedarfsorientiert für einkommensschwache Haushalte (CAIF) und marktförmig für Haushalte mit mittlerem bis hohem Einkommen. Für die vier- bis fünfjährigen Kinder weisen beide Länder nahezu einen universellen Versorgungsgrad auf, mit einer hohen DeFamiliarisierung im Falle Uruguays und einer mittleren De-Familiarisierung in Chile. Bezüglich Geschlechtergerechtigkeit weist Chile eine leicht maternalistische Ausrichtung auf; in Uruguay übernimmt der Staat Mitverantwortung aufgrund des umfangreichen staatlichen Betreuungsagebots. Für die vier bis fünfjährigen Kinder sorgt Chile für grössere soziale Gerechtigkeit im Hinblick auf den Zugang zu ausserhäuslicher Betreuung, die dem bedarforientierten Typus (untere Schichten) und dem marktförmigen Typus (mittlere und obere Schichten) entspricht. Uruguay nähert sich grösserer sozialer Gerechtigkeit eher in einer staatsbürgerlichen Form an, mit umfangreichem staatlichen Angebot und begrenztem Privatangebot.

Die Hauptdifferenz bei der unbezahlten Hausarbeit besteht darin, dass diese in Uruguay verbreiteter ist als in Chile. Somit weist Chile ein Modell mit geringer Familiarisierung und Uruguay eines mit mittlerer Familiarisierung auf. Da es keine spezifische sozialpolitische Massnahme in diesem Bereich gibt und weil vorderhand Frauen diese Aufgaben übernehmen, ist die Geschlechtergerechtigkeit maternalistischer Prägung. Die soziale Gerechtigkeit kann keinem spezifischen Typus zugeordnet werden, jedoch ist eine regressive Verteilung zu beobachten, da die unbezahlte Hausarbeit bei Haushalten abnimmt, bei denen das Einkommen höher ist. Die bezahlte häusliche Versorgung ist in beiden Ländern ähnlich und begünstigt Familialismus.

Die Bereitstellung findet auf dem Markt statt, mit einer leicht maternalistischen Geschlechtergerechtigkeit, die auf die gesetzlichen Fortschritte zugunsten der Arbeitsrechte von Haushaltshilfen zurückgeht. Der soziale Gerechtigkeitstypus ist insofern kontributiv, als die erteilten Rechte vom Beschäftigungsstatus abhängen. Gleichzeitig ist eine regressive Verteilung der bezahlten Hausarbeit zu beobachten, weil deren Nachfrage mehrheitlich aus Haushalten der mittleren und oberen Schichten kommt, während sie von Frauen der unteren Schichten und unteren Mittelschichten selbst ausgeführt wird. 


\begin{tabular}{|c|c|c|c|c|c|}
\hline Art der Versorgung & Land & Familialismus & Bereich & Geschlechtergerechtigkeit & $\begin{array}{l}\text { Soziale Gerech- } \\
\text { tigkeit }\end{array}$ \\
\hline \multirow[t]{2}{*}{$\begin{array}{l}\text { Ausserhäusliche Betreu- } \\
\text { ungsleistungen (0-3 Jahre) }\end{array}$} & Chile & $\begin{array}{l}\text { geringe De- } \\
\text { Familiarisierung }\end{array}$ & $\begin{array}{l}\text { Staat }(=) \\
\text { Markt }(=)\end{array}$ & $\begin{array}{l}\text { gering, da traditionale Arbeits- } \\
\text { teilung: Maternalismus }\end{array}$ & $\begin{array}{c}\text { segmentiert: } \\
\text { - bedarfsorientiert } \\
\text { (staatl.) } \\
\text { - kontributiv } \\
\text { - marktförmig } \\
\text { (privat) }\end{array}$ \\
\hline & Uruguay & $\begin{array}{l}\text { geringe De- } \\
\text { Familiarisierung }\end{array}$ & $\begin{array}{c}\text { Markt (+) } \\
\text { Staat (-) }\end{array}$ & $\begin{array}{l}\text { gering, da traditionale Arbeits- } \\
\text { teilung: Maternalismus }\end{array}$ & $\begin{array}{c}\text { segmentiert: } \\
\text { - bedarfsorientiert } \\
\text { (staatl.) } \\
\text { - marktförmig } \\
\text { (privat) }\end{array}$ \\
\hline \multirow[t]{2}{*}{$\begin{array}{l}\text { Ausserhäusliche Betreu- } \\
\text { ungsleistungen (4-5 Jahre) }\end{array}$} & Chile & $\begin{array}{l}\text { mittlere De- } \\
\text { Familiarisierung }\end{array}$ & $\begin{array}{l}\text { Staat }(=) \\
\text { Markt }(=)\end{array}$ & $\begin{array}{c}\text { mittel, da gering ausgeprägter } \\
\text { Maternalismus }\end{array}$ & $\begin{array}{c}\text { segmentiert: } \\
\text { - bedarfsorientiert } \\
\text { (staatl.) } \\
\text { - marktförmig } \\
\text { (privat) }\end{array}$ \\
\hline & Uruguay & $\begin{array}{c}\text { starke De- } \\
\text { Familiarisierung }\end{array}$ & $\begin{array}{l}\text { Staat }(+) \\
\text { Markt (-) }\end{array}$ & $\begin{array}{c}\text { hoch, da staatliche Mitverant- } \\
\text { wortung }\end{array}$ & $\begin{array}{c}\text { egalitäre Tendenz } \\
\text { - staatsbürgerlich } \\
\text { (staatl.) } \\
\text { - marktförmig } \\
\text { (privat) }\end{array}$ \\
\hline \multirow[t]{2}{*}{$\begin{array}{l}\text { Unbezahlte häusliche Be- } \\
\text { treuungsleistungen }\end{array}$} & Chile & $\begin{array}{l}\text { geringe Familiari- } \\
\text { sierung }\end{array}$ & Familie & $\begin{array}{l}\text { gering, da traditionale Arbeits- } \\
\text { teilung: Maternalismus }\end{array}$ & $\begin{array}{c}\text { (regressive Vertei- } \\
\text { lung) }\end{array}$ \\
\hline & Uruguay & $\begin{array}{l}\text { mittlere Familiari- } \\
\text { sierung }\end{array}$ & Familie & $\begin{array}{c}\text { gering, da traditionale Arbeits- } \\
\text { teilung: Maternalismus }\end{array}$ & $\begin{array}{c}\text { (regressive Vertei- } \\
\text { lung) }\end{array}$ \\
\hline \multirow[t]{2}{*}{$\begin{array}{l}\text { Bezahlte häusliche Betreu- } \\
\text { ungsleistungen }\end{array}$} & Chile & $\begin{array}{l}\text { geringe De- } \\
\text { Familiarisierung }\end{array}$ & Markt & $\begin{array}{c}\text { mittel, da gering ausgeprägter } \\
\text { Maternalismus }\end{array}$ & $\begin{array}{c}\text { Kontributiv } \\
\text { (regressive Vertei- } \\
\text { lung) }\end{array}$ \\
\hline & Uruguay & $\begin{array}{l}\text { geringe De- } \\
\text { Familiarisierung }\end{array}$ & Markt & $\begin{array}{c}\text { mittel, da gering ausgeprägter } \\
\text { Maternalismus }\end{array}$ & $\begin{array}{c}\text { kontributiv } \\
\text { (regressive Vertei- } \\
\text { lung) }\end{array}$ \\
\hline \multirow[t]{2}{*}{ Betreuungszeit } & Chile & $\begin{array}{l}\text { Mittlere Familiari- } \\
\text { sierung }\end{array}$ & Staat & $\begin{array}{l}\text { gering, da traditionale Arbeits- } \\
\text { teilung: Maternalismus }\end{array}$ & kontributiv \\
\hline & Uruguay & $\begin{array}{l}\text { Mittlere Familiari- } \\
\text { sierung }\end{array}$ & Staat & $\begin{array}{c}\text { mittel, da gering ausgeprägter } \\
\text { Maternalismus }\end{array}$ & kontributiv \\
\hline
\end{tabular}

Tabelle 2: Vergleich der Kinderbetreuungsmodelle (0-5 Jahre) zwischen Chile und Uruguay, 2013. Quelle: Eigene Darstellung. 
Bei der Betreuungszeit weisen beide Länder einen leichten Familialismus auf und zwar in dem Masse, wie die Gesetzgebung den Schutz des Arbeitsplatzes von beschäftigten Müttern und Vätern gewährt. Obwohl diese Freistellung die Care-Funktion zurück in den familiären Bereich (Familialismus) verlagert, schützt sie gleichzeitig die bezahlte Arbeit (vor allem die der Frauen), indem die wirtschaftliche Autonomie gestärkt und die produktiven und reproduktiven Rollen vereinbart werden (leicht). In beiden Fällen ist der Staat der Hauptversorger, da er die Freistellungen finanziert. Bezüglich Geschlechtergerechtigkeit weist Chile ein maternalistisches Profil auf, da die Differenzen in Bezug auf die Betreuungszeit zwischen Frauen und Männern grösser sind als in Uruguay. Dieses Land zeichnet sich hingegen durch einen leichten Maternalismus aus, weil Männern mehr Zeit für die Übernahme dieser Aufgaben zur Verfügung steht. Die soziale Gerechtigkeit hat in beiden Fällen einen kontributiven Charakter, weil es zumeist die Lohnabhängigen sind, die Zugang zu diesen Freistellungen haben. Obwohl diese Freistellung in beiden Ländern auf selbständig Erwerbende erweitert wurde, nimmt diese Beschäftigungsgruppe das Angebot bis jetzt nur begrenzt in Anspruch (Batthyány et al., 2015; SUCESO, 2015).

Zusammenfassend lässt sich attestieren, dass beide Länder aufgrund der kürzlich eingeleiteten Reformen eine Spannung zwischen Familialismus und optionalem Familialismus aufweisen. In beiden Ländern ist die ausserhäusliche Versorgung für Kleinkinder stark familiarisiert und für ältere Kinder eher de-familiarisiert. Chile wahrt seinen liberal-marktorientierten Charakter und Uruguay ein staatsbürgerlich-staatliches Profil. Die häusliche Versorgung ist sich in beiden analysierten Fällen sehr ähnlich. In Bezug auf die Betreuungszeiten weist Chile einen maternalistisch geprägten Charakter auf, was auf der Mehrzeit beruht, die den Frauen gegenüber den Männern zur Verfügung steht. Uruguay hingegen fördert eine stärkere Beteiligung der Männer, indem ihnen mehr Zeit bei der Inanspruchnahme dieser Freistellungen gewährt wird. Der Übergang des Kinderbetreuungsmodells von einem traditionellen Familialismus hin zu einem optionalen Familialismus kann beim Ausbleiben von sozialpolitischen Massnahmen eine ambivalente Form annehmen: Während die Anzahl derjenigen Frauen abnimmt, die sich ausschliesslich Care-Arbeiten widmen, wächst gleichzeitig die Anzahl erwerbstätiger Frauen, die jedoch weiterhin Hauptverantwortliche für die Betreuung zu Hause sind.

\section{Literatur}

Batthyány, Karina (2015). Las políticas y el cuidado en América Latina. Una mirada a las experiencias regionales. Asuntos de Género. 124. Santiago de Chile: CEPAL/Cooperación Española.

Batthyány, Karina, Genta, Natalia, und Perrotta, Valentina (2015). Avanzando hacia la corresponsabilidad en los cuidados. Análisis de las licencias parentales en el Uruguay. 128. Santiago de Chile: CEPAL.

Blofield, Merike, und Martínez Franzoni, Juliana (2015). Maternalism, Co-responsibility and Social Equity: a typology of work-family policies. Social Politics, 22(1), 38-59.

CEPAL (2015). CEPALSTAT. Zugriff am 16.11.2015, auf http://estadisticas.cepal.org. 
Daly, Mary, und Lewis, Jane (2011). El concepto de "social care" y el análisis de los Estados de Bienestar contemporáneos. In Cristina Carrasco, Cristina Borderías \& Teresa Torns (Eds.), El trabajo de cuidados. Historia, teoría y políticas (2011 ed., pp. 225-251). Madrid: Catarata.

DT (2015). Código del Trabajo. Santiago de Chile: Dirección del Trabajo.

Esping-Andersen, Gøsta (1990). The three worlds of the welfare capitalism (2015 ed.). Cambridge: Polity Press.

Esping-Andersen, Gøsta (2000). Fundamentos sociales de las economías postindustriales. Barcelona: Ariel.

INE (2013). Indicadores demográficos. Zugriff am 16.11.2015, auf http://www.ine.gub.uy

INE (2015). Proyecciones de población 2014. Santiago de Chile: Instituto Nacional de Estadística, Chile.

Leitner, Sigrid (2003). Varieties of familialism: The caring function of the family in comparative perspective. European Societies, 5(4), 353-375.

Lupica, Carina (2015). Corresponsabilidad de los cuidados y autonomía económica de las mujeres. Lecciones aprendidas del permiso postnatal en Chile. Asuntos de Género. 126. Santiago de Chile: CEPAL/Ministerio de Asuntos Exteriores de Noruega.

MIDEPLAN (2006). Ley N² 20.379. Ministerio de Planificación, Chile.

MIDES (2014). Cuidados como sistema. Propuesta para un modelo solidario y corresponsabilidad de cuidados en Uruguay. Montevideo: Ministerio de Desarrollo Social.

MINTRAB (2014). Ley 20.786. Valparaíso, Chile: Biblioteca del Congreso Nacional de Chile.

MTSS (2006). Ley No 18.065. Trabajo Doméstico. Normas para su regulación. Montevideo: Ministerio del Trabajo y Seguridad Social. Zugriff am 12.01.2016, auf http://www.parlamento.gub.uy.

MTSS (2013). Ley $N^{o}$ 19.16. Subsidios por maternidad y por paternidad para trabajadores de la actividad privada. Montevideo: Ministerio de Trabajo y Seguridad Social. Zugriff am 20.01.2016, auf http://www.bps.gub.uy.

SUCESO (2015). Subsidio por permiso postnatal parental 2011-2014. Santiago de Chile: Superintendencia de Seguridad Social.

Staab, Silke (2013). Protección social para la infancia y adolescencia en Chile. 180. Santiago de Chile: CEPAL/UNICEF.

Ullmann, Heidi, Maldonado Valera, Carlos, und Nieves Rico, María (2014). La evolución de las estructuras familiares en América Latina, 1990-2010. Los retos de la pobreza, la vulnerabilidad y el cuidado. Políticas Sociales. 193. Santiago de Chile: CEPAL/UNICEF. 